\title{
An Analysis of the Dressing Features of Elderly Women in Contemporary Cities of Hubei Province
}

\author{
Jun $\mathrm{YIN}^{1, \text { a }}$, Dan $\mathrm{ZHOU}^{2, \mathrm{~b}}$ * \\ ${ }^{1}$ Wuhan Textile University, Wuhan, 430073, China \\ ${ }^{2}$ School of Foreign Languages, Wuhan Textile University, Wuhan, 430073, China \\ aemail: 12408113@qq.com, bemail:zhoudan@wtu.edu.cn
}

Key words: dressing; Hubei Province; the urban elderly

\begin{abstract}
Based on the fact-finding of the elderly women in the three cities of Hubei Province, this paper clearly reveals their dressing features. The results indicate that: 1 . The elderly are more subdivided in contemporary cities of Hubei. Though the traditional ethics, the aesthetic value and the consumption idea decide the general dressing features like "simplification" and "following the trend", they still have some obvious individual differences owing to their different educational and income level, personal beliefs and regional disparity; 2. Among the six motives of the elderly dressing, "to meet the self-perception" has become the primary one. More elderly transfer their attention from details to their overall looks; 3. The dressing style of elderly women can be divided into urban fashion style, ethnic style, simple and traditional style which all obey the same principle -- fabric and comfort.
\end{abstract}

\section{Introduction}

According to statistics, at the end of 2015, the population of the elderly who are older than 60 years old amounts to 22.2 million, accounting for $16.1 \%$ of the total population, which shows that China has entered the ultra-aging society. The surge in the elderly population has a huge impact on national and social planning, government policy-making as well as adjustment of industrial structure. Scholars have conducted many studies on their care policy, living conditions, diseases, nursing care and so on. However, few have attached great importance to the elderly culture, mental health and consumer behavior. Thus, a systematical study on it is academic value in sociology, anthropology and culturology. [1] In terms of mass consumption society, in-depth analysis of its behavior is conducive to more accurate positioning of the target market.

In this study, we study the phenomenon of the dressing of elderly women in Wuhan, Yichang and Xiangyang of Hubei province, through field research, interview and questionnaire to acquire their dressing idea and dressing style. [2]By use of SPSS tool to collect and analyze data, and then combined with behavior science, psychology of aging and clothing aesthetic theory, attempt to find out the dressing features of elderly women in contemporary era.

\section{The Subdivision of the Elderly}

The main focus of the survey is the urban elderly women whose ages range from 55 to 70 years old (after retirement). Through comparing their age, physiological status and other basic information, significant differences of times are found in their physical and mental health.

The first group (age from 55 to 60 years old) have relatively good physical quality and can move flexibly. They are relatively young, accounting for about $47 \%$ of the total elderly. This group's education background is mainly at least junior high school, and they just retire with relatively strong economic strength. Some people often participate in various social and cultural activities or some social part-time jobs, belonging to the groups with larger circle of life and more positive and optimistic personality. [3] They have high learning ability and perception, are willing to try new things and share a variety of information. They can use modern communication tools 
with facility.

The second group (age from 61 to 65 years old) have fair physical quality, their motor system function weakens, respiratory function decreases and physiological function degrades especially the vision. The proportion of this group is about 39\%. This elderly group has formed a certain cultural and entertainment circles, but shows a lower interest in learning new things. They gradually withdraw from social part-time jobs.

The third group (age from 66 to 70 years old) have more physical pain, the body's immune function significantly decreases, with gradually slow action and response, also visual loss. The proportion of this group is about $14 \%$. Most of these elderly people are not involved in social part-time jobs. [4]They mainly concern about health, and are willing to participate in the discussion of health topics. However, they have fair economic conditions and show little interest in learning to use new things and new tools.

\section{Dressing Features and Dressing Ideas of the Elderly}

\section{A. The Common Features and Individual Differences of Dressing}

Through the interview survey, it was found that the dressing behavior of the elderly women in the three cities of Hubei showed common features and individual differences.

Affected by the Chinese traditional ethics, aesthetic values, consumption concept and the lagging development of the elderly clothing market, 55-70-year-old urban women's dressing share the following three common features: (1) The practical function "to keep warm" is no longer the primary motive for elderly women to choose dressing, but the aesthetic function has gradually become a consideration; (2) the aesthetic standard of dressing in the current stage is still "simple and proper is beauty". The elderly women over the age of 55 are deeply influenced by the traditional ethics in the aesthetic choice of dressing. "Simple and proper" is still the principle that most elderly women choose dressing, particularly evident among the 65-70-year-old women; (3) "others' opinions" have a considerable weight in the choice of dressing. In this survey, elderly women involved in group activities take up more than 63\%. In group activities, the dressing remarks from friends or teammates become an important criterion for elderly women's dressing.

According to the group division of the elderly women, although there are some common features of their dressing, but there are also some significant individual differences because of different region, culture, occupation and post-retirement life. The differences mainly lie in the following two aspects: (1) there are differences in the number and aesthetic preference of elderly women in provincial and prefecture-level cities. The elderly women in Wuhan change their coat about once a day in spring and summer, about once a week in autumn and winter, and their dressing style is more diversified; while elderly women in Yichang and Xiangyang change their coat about three times a week in spring and summer, about twice a week in autumn and winter, and their dressing style is more plain. (2) Educational and professional background lead to the different aesthetic standards. The elderly women who are highly educated and work as teachers, doctors, literary and art workers before retirement usually have more diversified dressing style, "urban", "classic", "ethnic" style, for example, have become their choices. Also, the elderly who frequently take part in group activities after retirement emphasize more on the quality of dressing.

\section{B. The Dressing Ideas of the Elderly}

The Theory of Rational Behavior (TRA) argues that the general concept of the individual and the society in which he lives is an important determinant of one's attitudes and values. And personal attitudes and values determine whether a person will have the motivation to take a particular act, the act finally decides whether this behavior will be adopted by a person. The concept of social public aesthetics has always had an important impact on the formation of the aesthetic ideas of the Chinese elderly, and personal experience and value orientation have become an important motive for her / him whether he has adopted this dressing style or not. In recent years, the awareness of social 
collectivism in China has changed, which affect the consumer values and dressing ideas of the elderly.

First of all, the dressing motive has turned to self-experience. Through Analysis of the importance scale and main factor of dressing target, the order of the elderly women's dressing motives are as follows: (1) I think it is beautiful and comfortable to wear (to pursue physical \& mental comfort and appearance); (2) I think it can represent my personal style (to create a self-image tool); (3) I think the occasion is appropriate (to seek expression of rituals); (4) Everyone thinks it is good-looking (to seek group approval); (5) I think it is fashionable (follow the novelty); (6) I think it matches with my identity (to show the identity and status).

Second, the awareness of overall dressing is highlighted. More elderly transfer their attention from details to their overall looks. $57 \%$ of the respondents believe that the overall coordination is more important than a single dressing detail. The overall coordination mainly refers to the color coordination, appropriate style, fit version type and comfortable fabric. But in daily dressing, there is only $13 \%$ of the elderly pay attention to the entire looks, the main accessories include shoes, scarves, bags, glasses and jewelry.

Table 1 Analysis of the importance scale and main factor of dressing target

\begin{tabular}{|l|c|}
\hline \multicolumn{1}{|c|}{$\begin{array}{c}\text { The variable names and questions for } \\
\text { dressing motives }\end{array}$} & Average value \\
\hline I think it is fashionable. & 2.77 \\
\hline Everyone thinks it is good-looking. & 2.94 \\
\hline I think the occasion is appropriate. & 3.09 \\
\hline I think it can represent my personal style. & 3.21 \\
\hline I think it is beautiful and comfortable to wear. & 3.66 \\
\hline I think it matches with my identity. & 2.03 \\
\hline
\end{tabular}

\section{Dressing Styles of the Elderly}

With the rapid development of information and communication technology, it is more convenient for the elderly to access to the information of dressing, which directly lead to the diversification of the elderly dressing styles. Among the seven mentioned dressing styles in the questionnaire, the elderly understanding of them sorted as follows: sports and leisure, national customs, urban fashion, traditional and classic, gentle and graceful, rural and pastoral, rock street; the elderly barely understand the rock street style. And 58\% of the elderly understand four or more dressing style, $22 \%$ understand three styles, $9 \%$ understand two styles and $11 \%$ do not understand dressing style at all. In their daily dressing, $2 \%$ of the elderly have tried $4-5$ kinds of styles, $57 \%$ have tried 3 kinds; 38\% have tried 2 kinds and 3\% have tried only 1 kind of the styles.

\section{A. Different Kinds of Dressing Styles}

Through the classification analysis, currently the dressing style of the elderly in Hubei can be divided into three types: urban fashion, ethnic and simple traditional.

Urban fashion dressing style mainly includes: urban fashion and graceful these two styles, embodied in its young and fashionable design, color and details, which are integrated with the urban pop culture. The elderly fond of this style are more familiar with the popular culture, willing to exchange ideas with young people, have access to clothing information from the multi-channel, and go shopping both on-line and in physical stores, concerned about the clothing brand. Of them, $42 \%$ of the elderly retired from the occupations related to culture, education or business management. They like urban life have a wide range of hobbies and participate in social groups activities.

Ethnic dressing style is embodied in the clothing colors, patterns, fabric accessories and other details which are from minority costumes. Its vivid color with strong contrast and dressing patterns like national flowers, national totem makes this kind of dressing novel. The elderly fond of this 
style usually have a foundation of minority culture, they are lively and outgoing, participate in square dancing or other recreational activities. Of them, $61 \%$ of the elderly retired from the occupations related to literature and art, cultural study or design.

Simple traditional dressing style mainly includes: traditional and classic, rural and pastoral, embodied in the basic design and less details. The color of clothing is conservative, usually dark colors. The clothing is made of cotton and flax. The elderly fond of this style usually live a simple life and emphasize on Chinese traditional values and ethics. Of them, $57 \%$ of the elderly retired from the occupations like workers or clerks.

\section{B. Causes of Different Dressing Styles}

Among the 187 valid questionnaires, urban fashion style accounted for $26 \%$, ethic style accounted for $21 \%$, and simple traditional style accounted for 53\%. It shows that despite the openness of ideas in the information age, the vast majority of contemporary Chinese elderly tend to be conservative in dressing.

The causes of this result can be concluded as follows: (1) The traditional concept is far-reaching. Although the elderly dressing motive tends to improve self-satisfaction, but they are still deeply influenced by the Chinese traditional ethics: "not be too fancy", "not be too outrageous", "we say good is really good" still have a profound impact on the majority of elderly women; (2) Product supply cannot meet the diversity of consumer demand. From the perspective of the market, the clothing brand for elderly is relatively less than other age groups. The information on elderly dressing fashion is less as well. There seems to be no magazine about the elderly fashion. Although there are $1 / 4$ of the elderly women have a preference for the urban fashion style, they lack professional guidance in actual life. Furthermore, the homogenization of product is serious: most dressing shares similar styles and patterns, because the clothing products lack professional design based on the physiological characteristics and aesthetic psychology of the elderly.

In this study, we study the phenomenon of the dressing of elderly women in Wuhan, Yi chang and Xiang yang of Hubei province through field research, interview and questionnaire to acquire their dressing idea and dressing style. Through the long-term close attention to the dressing of the elderly women, the formation of its consumer culture can be easily understood, and the consumer psychology and behavior trends can be grasped for the upcoming aging consumer market.

\section{Acknowledgement}

In this paper, the research was sponsored by Project of Humanities and Social Science of Hubei Province (Project No. 17Y057), Foundation of Hubei Educational Committee(Project No. 2016322) , Foundation of Hubei Educational Committee(Project No. 2016GB036)and Foundation of Wuhan Textile University (Project No. 163092; No. 163053).

\section{References}

[1] Ministry of Civil Affairs "2015 Social Service Development Statistics Bulletin", 2016(07)

[2] Liu Chun, Yang Zhiliang. Study on Dressing Motives[J]. Journal of Applied Psychology. 1996, 2(1), 17-22

[3] Wang Kunliang. Study on the Lifestyle and Apparel Consumption Concept of the Elderly in the South Area [J]. Chemical Fiber and Textile Technology. 2007, 06, 47-49

[4] Wang Fei. Empirical Study on Consumer Behavior of Urban Elderly in China [J]. Population and Development. 2015, 21(3), 101-11 